\title{
Ecdysone Elicits Chronic Renal Impairment via Mineralocorticoid-Like Pathogenic Activities
}

\author{
Minglei Lu ${ }^{a, b, c}$ Pei Wang ${ }^{a}$ Sijie Zhou ${ }^{a, c}$ Bryce Flickinger ${ }^{b, d} \quad$ Deepak Malhotra ${ }^{b}$ \\ Yan Ge $\mathrm{G}^{\mathrm{b}, \mathrm{c}}$ Marc Tatare Lance Dworkin ${ }^{\mathrm{b}}$ Zhangsuo Liu ${ }^{\mathrm{a}}$ Rujun Gonga,b,c \\ Institute of Nephrology, Department of Nephrology, the First Affiliated Hospital of Zhengzhou \\ University, Zhengzhou, China, 'Division of Nephrology, Department of Medicine, University of Toledo \\ College of Medicine, Toledo, 'Division of Kidney Disease and Hypertension, Brown University School of \\ Medicine, Providence, 'Denison University, Granville, e'Department of Ecology and Evolutionary Biology, \\ Brown University, Providence, USA
}

\section{Key Words}

Ecdysone $\bullet$ Kidney $\bullet$ Mineralocorticoid receptor $\bullet$ Inner medullar collecting duct cell

\begin{abstract}
Background/Aims: Ecdysteroids are steroidal insect molting hormones that also exist in herbs. Ecdysteroid-containing adaptogens have been popularly used to improve well-being and by bodybuilders for muscle growth. However, the use of ecdysone in mammals is also associated with kidney growth and enlargement, indications of disturbed kidney homeostasis. The underlying pathogenic mechanism remains to be clarified. Methods: Virtual screening tools were employed to identify compounds that are homologous to ecdysone and to predict putative ecdysone-interacting proteins. The kidney effect of ecdysone was examined in vitro and in vivo and compared with that of aldosterone. Cellular apoptosis was estimated by terminal deoxynucleotidyl transferase dUTP nick end labeling. Cell motility was assessed by scratch-wound cell migration assay. Blood urea nitrogen was measured to evaluate renal function. Western immunblot analysis was employed to determine the expression profile of interested proteins. Results: Computational molecular structure analysis revealed that ecdysone is highly homologous to aldosterone. Moreover, virtual screening based on compound-protein interaction profiles identified the Mineralocorticoid Receptor (MR) to potentially interact with ecdysone. Accordingly, to assess potential biological functions of ecdysone in mammals, ecdysone was applied to mineralocorticoid-sensitive inner medullar collecting duct cells. Ecdysone induced mesenchymal accumulation of extracellular matrix and epithelial dedifferentiation characterized by de novo expression of $\alpha$-smooth muscle actin. In addition, ecdysone elicited cellular apoptosis and retarded cell motility, akin to the effect of aldosterone. In vivo, daily treatment of mice with ecdysone increased cell apoptosis in the kidney, impaired renal function and elicited early signs of renal fibrogenesis, marked by deposition
\end{abstract}

\begin{tabular}{ll}
\hline Zhangsuo Liu & Institute of Nephrology, The First Affiliated Hospital of Zhengzhou University, Zhengzhou (China); \\
and Rujun Gong & College of Medicine, University of Toledo, Toledo (USA) \\
& E-Mail zhangsuoliu@sina.com; Rujun.Gong@UToledo.edu
\end{tabular}


of collagen and fibronectin in tubulointerstitium, reminiscent of the action of aldosterone. The MR signaling pathway is likely responsible for the cellular and pathobiological effects of ecdysone, as evidenced by strong ecdysone-induced MR nuclear translocation in renal tubular cells both in vitro and in vivo, while blockade of MR by concomitant spironolactone treatment largely abolished the detrimental effects of ecdysone. Conclusion: Our findings suggest that ecdysone induces mineralocorticoid-dependent activities that impair renal function and elicit renal injury.

(C) 2018 The Author(s)

Published by S. Karger AG, Basel

\section{Introduction}

Complementary and alternative medical practices often prescribe natural medications with undetermined pharmacologic properties, such as herbs, minerals, insect- or animalderived products and adaptogens, to help the body adapt to stress and to treat human diseases [1]. So-called adaptogens can contain ecdysteroids - steroidal insect molting hormones that also exist in herbs [2-5]. Ecdysteroid-containing adaptogens have been popularly used for improving well-being and by bodybuilders for muscle growth [6-8]. In stark contrast to the traditional opinion that natural medications used by alternative medical practices are largely safe and beneficial by exerting a normalizing effect upon bodily processes, a growing body of evidence recently suggests that a number of natural medications might be detrimental to kidney health [6, 9-11]. In particular, ecdysone targets the drosophila Malpighian tubules, which are equivalent to renal tubules in mammals, and regulates ionic changes and water retention in arthropods [12,13]. Recently, the use of ecdysone-containing adaptogens in mammalian has been associated with kidney growth and enlargement [14-16], which are evident clinical signs of disturbed kidney homeostasis. The mechanism underlying this renal side effect of ecdysone remains to be defined.

The steroid hormone ecdysone is the central regulator of insect developmental transitions [17]. It is a steroidal prohormone of the major insect molting hormone 20-hydroxyecdysone, which is secreted from the prothoracic glands $[18,19]$. In arthropods like Drosophila melanogaster, ecdysone functions via ecdysone receptor (EcR), a member of the nuclear receptor superfamily, to induce metamorphosis [20,21]. Because ecdysteroids are apparently not endogenously generated components of mammalian systems and mammalian cells do not express the insect EcR, it has long been believed that ecdysone has no activities on mammals. As such, this has been subsequently harnessed to create the ecdysone-inducible gene switch control system in mammalian cells and transgenic mice to modulate transgene expression [22]. However, burgeoning evidence proves that ecdysone does have effect in mammals and humans [23]. In human immunocompetent cells like lymphocytes, ecdysteroids are able to induce E-rosette formation and activation [24]. In vivo, in both experimental animals and humans, ecdysone has been reported to induce a constellation of physiologic effects on protein, lipid and carbohydrate metabolisms and in diverse organ systems, including the muscle, liver, skin, brain and kidney $[23,25,26]$. The molecular mechanism underlying these ecdysone activities in mammals is elusive, but may involve a mixture or cocktail effect that resembles multiple mammalian steroid hormones like glucocorticoids, mineralocorticoids or sex hormones. In support of this contention, ecdysone contains the common steroid core structure that is composed of seventeen carbon atoms, bonded in the parent four "fused" rings: three six-member cyclohexane rings and one five-member cyclopentane ring. Moreover, similar to mammalian steroid hormone system, 20-hydroxyecdysone (20E), the activated form of ecdysone in arthropods, also functions through binding to its cognate nuclear hormone receptor EcR $[21,27]$. Based on these observations, we speculate that ecdysone might exert a steroid-like effect in mammals by which it modulates renal pathobiology. To test this hypothesis, we used virtual screening tools to identify compounds that are homologous to ecdysone as well as the putative mammalian targets of ecdysone. We then assessed the function of ecdysone in mammals through analysis of in vitro cultured renal tubular cells and by in vivo treatment of mice with ecdysone.

\section{KARGER}




\section{Cellular Physiology Cell Physiol Biochem 2018;49:1633-1645 \begin{tabular}{ll|l} 
and Biochemistry Published online: 14 September, 2018 & $\begin{array}{l}\text { (c) } 2018 \text { The Author(s). Published by S. Karger AG, Basel } \\
\text { www.karger.com/cpb }\end{array}$ \\
\hline
\end{tabular}}

Lu et al.: Harmful Effects of Ecdysone on the Kidney

\section{Materials and Methods}

\section{In silico chemical similarity search and biological target identification}

Chemical similarity search was performed with SciFinder (https://origin-scifinder.cas.org/scifinder/). SciFinder uses the Tanimoto algorithm to compare substances in the Chemical Abstracts Service database with a query structure, here ecdysone. Similarity is based on a two-dimensional small molecule comparison using a Tanimoto similarity metric that is based on fingerprint-based similarity calculations. For in silico drug target predictions we used PharmMapper (http://lilab.ecust.edu.cn/pharmmapper/) to identify pharmacophore matches common to ecdysone and aldosterone. Molecular modeling of potential hormone binding receptors was performed with MOE (The Molecular Operating Environment) Version 2015.10. Three dimensional compound structure was modeled based on energy minimization using Amber 10:EHT force field. The crystal structure of mineralocorticoid receptor (MR, PDB ID: 2A3I) was modeled using the "QuickPrep" module with default protein parameters. Models of molecular docking applied the default Triangle Matcher placement method. The GBVI/WSA dG scoring function estimated the free energy of ligand binding.

\section{Cell culture and treatments}

Mouse inner medullary collecting duct cells (IMCD) were grown in DMEM/F12 media supplemented with 5\% Fetal Bovine Serum (FBS) and antibiotics (100U/ml penicillin and $100 \mu \mathrm{g} / \mathrm{ml}$ streptomycin). Cells were treated with: Aldosterone (Sigma-Aldrich, St. Louis, MO, USA), Ecdysone (Sigma-Aldrich), Spironolactone (Sigma-Aldrich) or Vehicle (dimethyl sulfoxide). All in vitro studies and immunoblot analyses were repeated three to four times.

\section{Cell migration assay}

Confluent monolayers of IMCD cells were scraped with a $10 \mu \mathrm{l}$ pipette tip. Phase contrast micrographs were obtained at 0,12 and 24h after scratching and quantified with Image J (v. 32, NIH, Bethesda, MD) to estimate area closure as the percent difference in wound area.

\section{Immunofluorescence staining}

Cultured IMCD cells were fixed and processed for fluorescent staining. Samples were stained with primary antibodies against MR (1:100, curtesy of Dr. Gomez-Sanchez, University of Mississippi) or fibronectin (1:100, Abcam) overnight at $4^{\circ} \mathrm{C}$ followed by Alexa Fluor-conjugated secondary antibodies (Invitrogen). As a negative control, primary antibodies were replaced by preimmune serum. Finally, samples were counterstained with propidium iodide, mounted with Vectashield mounting medium (Vector Laboratories, Burlingame, CA, USA) and visualized by a fluorescence microscope (BX43, Olympus, Tokyo,Japan). ImageJ software was used for post processing of the images (scaling, merging, and colocalization analysis).

\section{TdT-mediated dUTP nick end labeling (TUNEL) analysis}

After treatments, IMCD cells were fixed with 4\% paraformaldehyde phosphate-buffered saline and processed for staining with a TdT-mediated dUTP nick end labeling (TUNEL) kit (Promega, Madison, WI, USA). Ten random high-power microscopic fields were assessed for each treatment group. Results were presented as the percentage of TUNEL-positive cells of all cells.

\section{Animal Experiment Design}

Animal studies were approved by the Institutional Animal Care and Use Committee, and conform to the US Department of Agriculture regulations and the NIH's Guide for human care and use of Laboratory Animals. Male C57BL/ 6 mice aged 10 weeks were randomly assigned to 1 ) Vehicle control group ( $n=3)$ : mice were given vehicle (dimethyl sulfoxide, $0.1 \mathrm{ml}$ ) as daily subcutaneous injection; 2) Aldosterone group ( $\mathrm{n}=4)$ : mice received a daily subcutaneous injection of aldosterone at $6 \mu \mathrm{g} / \mathrm{g} / \mathrm{d} ; 3$ ) Aldosterone+Spironolactone group $(\mathrm{n}=4)$ : In addition to aldosterone, mice were given daily subcutaneous injection of spironolactone at $60 \mu \mathrm{g} / \mathrm{g} / \mathrm{d} ; 4)$ Ecdysone group ( $\mathrm{n}=4)$ : mice received a daily subcutaneous injection of ecdysone at $6 \mu \mathrm{g} / \mathrm{g} / \mathrm{d}$; 5) Ecdysone+Spironolactone group ( $\mathrm{n}=4)$ : In addition to ecdysone, mice were given a daily subcutaneous injection of spironolactone at $60 \mu \mathrm{g} / \mathrm{g} / \mathrm{d}$. After 14 days of treatment, mice were euthanized and blood and 


\section{Cellular Physiology Cell Physiol Biochem 2018;49:1633-1645

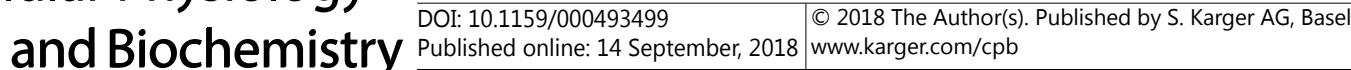

Lu et al.: Harmful Effects of Ecdysone on the Kidney

kidneys were collected for examinations. Blood urea nitrogen (BUN) concentration was measured by a urea assay kit (BioAssay Systems, Hayward, CA).

Renal Morphology Assessment and Immunohistochemistry Analysis

Paraffin-embedded kidneys were used to prepare into $4 \mu \mathrm{m}$ thick sections, deparaffinized and rehydrated. For general renal morphology assessment, the sections were subjected to Masson Trichrome staining. For immunohistochemistry, the sections were prepared for heat-induced epitope retrieval by using microwave. Peroxidase immunohistochemical staining was performed as described before [28] with primary antibodies against fibronectin (1:100, Abcam, San Francisco, CA, USA) or cleaved caspase-3 (1:100, Cell Signaling Technology, MA, USA). As a negative control, the primary antibody was replaced by nonimmune serum from the same species.

\section{Cellular and kidney nuclear protein extraction}

The cytoplasmic and nuclear fractions were prepared with the NE-PER kit (Thermo Scientific, Rockford, Illinois, USA) according to the manufacturer's instruction. Samples were processed for immunoblot analysis.

\section{Western Immunoblot Analysis}

Cultured cells were lysed and kidney tissues were homogenized in radioimmunoprecipitation assay buffer containing protease inhibitors. Samples were processed for immunoblot analysis as previously described [28]. Antibodies against the following molecules were used as primary antibodies to probe blots: cleaved caspase-3 (1:1000, Cell Signaling Technology, MA, USA), fibronectin (1:2000, Abcam, San Francisco, CA, USA), $\alpha$-SMA (1:1000, Abcam, San Francisco, CA, USA), collagen IV (1:500, Santa Cruz Biotechnology, Santa Cruz, CA), glyceraldehyde 3-phosphate dehydrogenase (GAPDH, 1:5000, Santa Cruz Biotechnology, Santa Cruz, CA). For immunoblot analysis, the integrated pixel density of bands was determined using the Image J software, version 1.48 (National Institutes of Health, Bethesda, MD).

\section{Statistical analyses}

Data are expressed as means \pm SD. Statistical analyses were performed using SPSS Statistics 17.0 software. For multiple comparisons of means between groups, statistical analysis was performed by oneway ANOVA, followed by the subsequent LSD test or Dunnett's T3 test. $P<0.05$ was considered statistically significant.

\section{Results}

Ecdysone is analogous to aldosterone in molecular structures and biological targets based on in silico analysis

To screen for substances that are similar to ecdysone in molecular structures, a similarity search was carried out with SciFinder [29], which employs the Tanimoto algorithm to compare all substances in the Chemical Abstracts Service (CAS) database with the structure query. Shown in Fig. 1a, the query of ecdysone with the CAS registry number 3604-87-3 resulted in an answer set of substances including 7-ketocholesterol, cerevisterol, aldosterone, and cholic acid, etc. Similarity rankings were based on a two-dimensional small molecule comparison using a Tanimoto similarity metric. Aldosterone with a Tanimoto score of 71 was among the top $10 \%$ substances similar to ecdysone.

To further affirm if ecdysone and aldosterone have similar or distinct drug target profiles, we applied the open-accessed PharmMapper server, a virtual screening tool for potential drug target identification using pharmacophore mapping approach [30]. Output targets include bile salt sulfotransferase, estradiol 17-beta-dehydrogenase 1, mineralocorticoid receptor (MR), and the vitamin D3 receptor, etc (Fig. 1b). Among these targets, MR (Protein Data Bank ID being 2A3I) was a common target that strongly fits both aldosterone and ecdysone (Fig. 1c). Molecular Operating Environment (MOE)-based 3D molecular modeling and simulations of protein-to-ligand interactions demonstrated that ecdysone and aldosterone target the same ligand binding domain (LBD) of MR (Fig. 1d). Collectively, these findings 
suggest that ecdysone may possess aldosterone-like activities and target MR.

Ecdysone elicits mesenchymal and profibrotic phenotypes in renal tubular cells

To ascertain if ecdysone has biological functions in a model of the mammalian renal system, we applied ecdysone to murine inner medullary collecting duct cells (IMCD), which are typical effector cells of aldosterone with prominent MR expression. Under basal condition with vehicle treatment, IMCD cells exhibited a typical cobblestone-like morphology of tubular epithelial cells (Fig. 2a). In contrast, both ecdysone and aldosterone treatments at a supraphysiologic $\left(10^{-7} \mathrm{M}\right)$ dose induced a branched spindle mesenchymallike cell shape within 24 or 48 h. As measured by immunoblot analysis, the expression of $\alpha$-smooth muscle actin (SMA), a standard mesenchymal marker, was induced after ecdysone treatment in a dose dependent fashion, as typically seen for aldosterone (Fig. 2b). Fibronectin, which is intimately involved in directing the organization of the interstitial extracellular matrix, was similarly increased in IMCD cells following aldosterone and ecdysone treatment, as assessed by fluorescent immunocytochemistry staining (Fig. 3a) and immunoblot analysis (Fig. 3b). These results are striking in part because $20 \mathrm{E}$ is the active form of ecdysteroid in insects, rather than ecdysone. We accordingly applied 20E to IMCD cells and assessed the suite of phenotypes $\left(10^{-7} \mathrm{M}, 10^{-9} \mathrm{M}\right)$ : $20 \mathrm{E}$ did not induce any effect upon cell morphology, $\alpha$-SMA or extracellular matrix (data not shown). This is in agreement with the results of in silico drug target

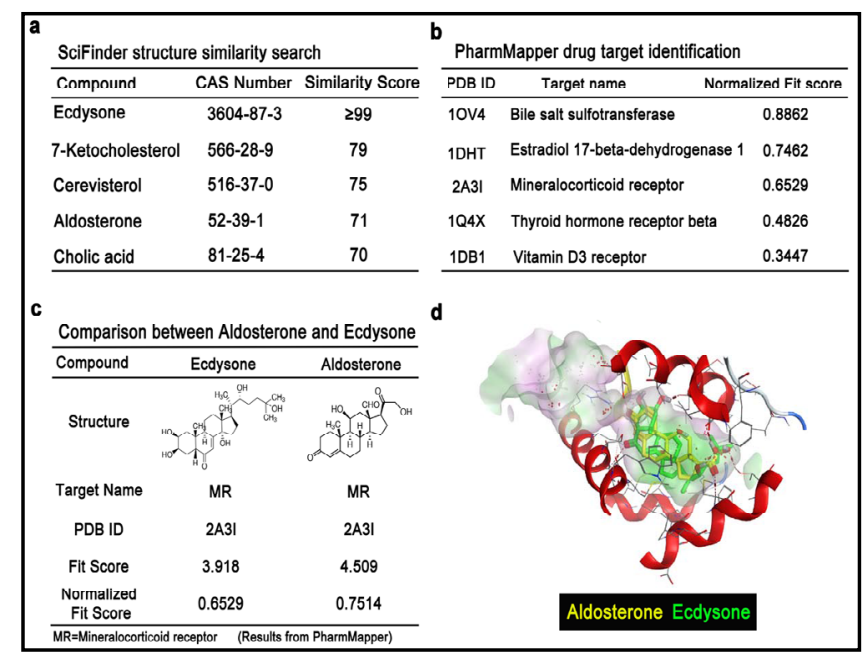

Fig. 1. In silico structural similarity search and drug target identification for ecdysone. (a) Selected high rank compounds similar to ecdysone based on Tanimoto similar score modeled by SciFinder, with Chemical Abstracts Service registry (CAS) number. (b) Selected high rank drug targets of ecdysone modeled by PharmMapper with Protein Data Bank identification (PDB ID) and normalized fit score. (c) MR (Protein Data Bank ID: 2A3I) is a common target that strongly fits both aldosterone and ecdysone based on the PharmMapper model. (d) Model of aldosterone (yellow) and ecdysone (green) binding to the mineralcorticoid receptor (2A3I) ligand binding domain, based on Molecular Operating Environment 3D molecular modeling simulation.

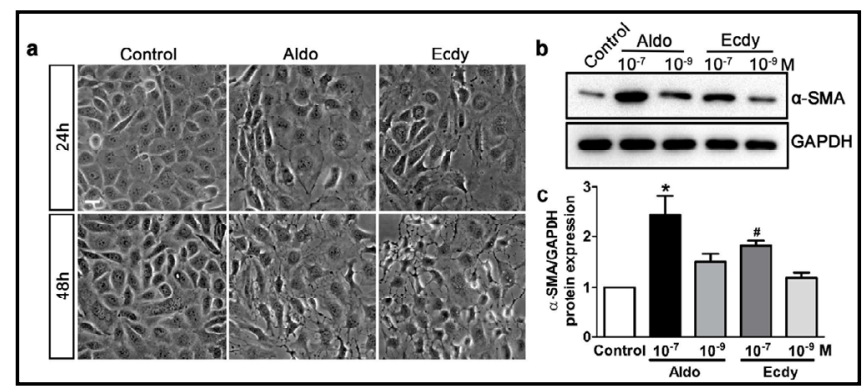

Fig. 2. Ecdysone induces aldosterone-like mesenchymal phenotypes in cultured renal tubular cells.(a) IMCD at 24 and 48 hours after treatment with aldosterone (Aldo, $10^{-7} \mathrm{M}$ ), ecdysone (Ecdy, $10^{-7} \mathrm{M}$ ) or vehicle control. Both aldosterone and ecdysone induced a branched spindle mesenchymal-like cell shape. Bar $=20 \mu \mathrm{m}$. (b) IMCD cells were treated with aldosterone, ecdysone at indicated concentrations or vehicle control for $48 \mathrm{~h}$ and processed for western blot for $\alpha$-smooth muscle actin (SMA) and glyceraldehyde 3-phosphate dehydrogenase (GAPDH). (c) Densitometric analysis of the relative levels of expression as normalized to GAPDH. ${ }^{*} \mathrm{P}=0.021$ vs control, ${ }^{\#} \mathrm{P}=0.002$ vs control $(n=4)$. 
a

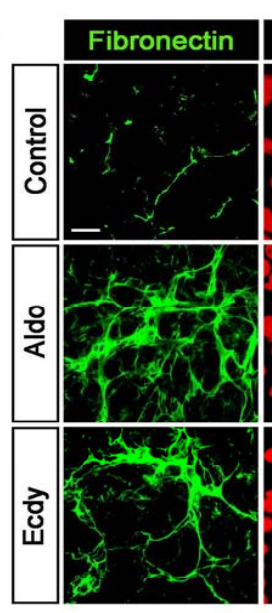

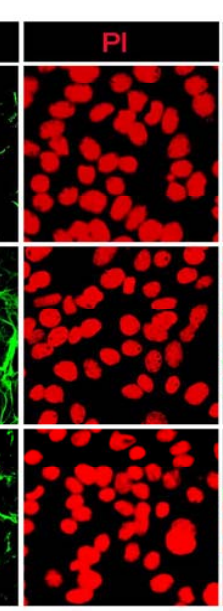
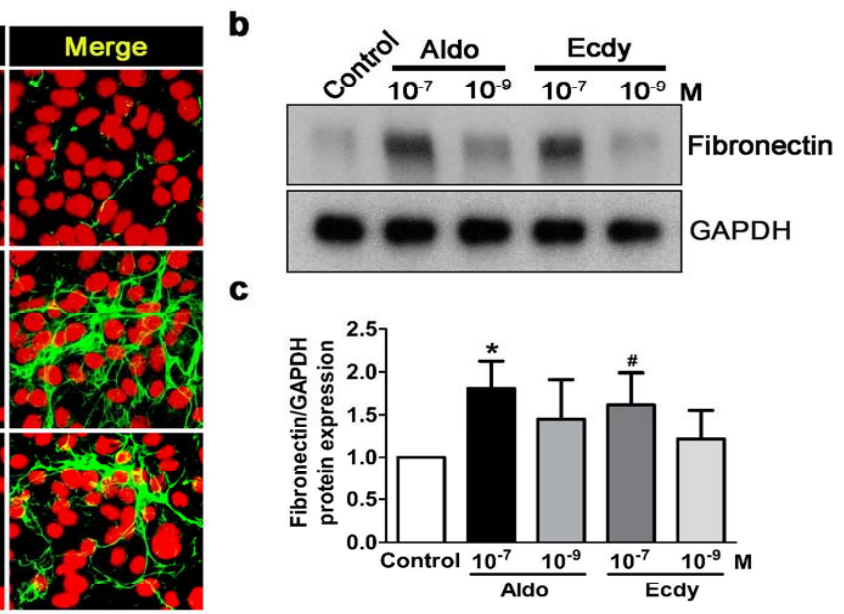

Fig. 3. Ecdysone induces extracellular matrix accumulation in IMCD cells. (a) Cells were treated as elaborated in Fig. 2. Cells were fixed at $48 \mathrm{~h}$ after indicated treatments and processed for fluorescence immunocytochemistry staining for fibronectin with propidium iodide (PI) counterstaining of nuclei. Bar $=20 \mu \mathrm{m}$. (b) Cell lysates were collected at $48 \mathrm{~h}$ and processed for western immunoblot analysis for indicated proteins, followed by densitometric analysis of the relative levels of expression as normalized to GAPDH. * $\mathrm{P}=0.014$ vs control, ${ }^{*} \mathrm{P}=0.048$ vs control $(\mathrm{n}=3)$.

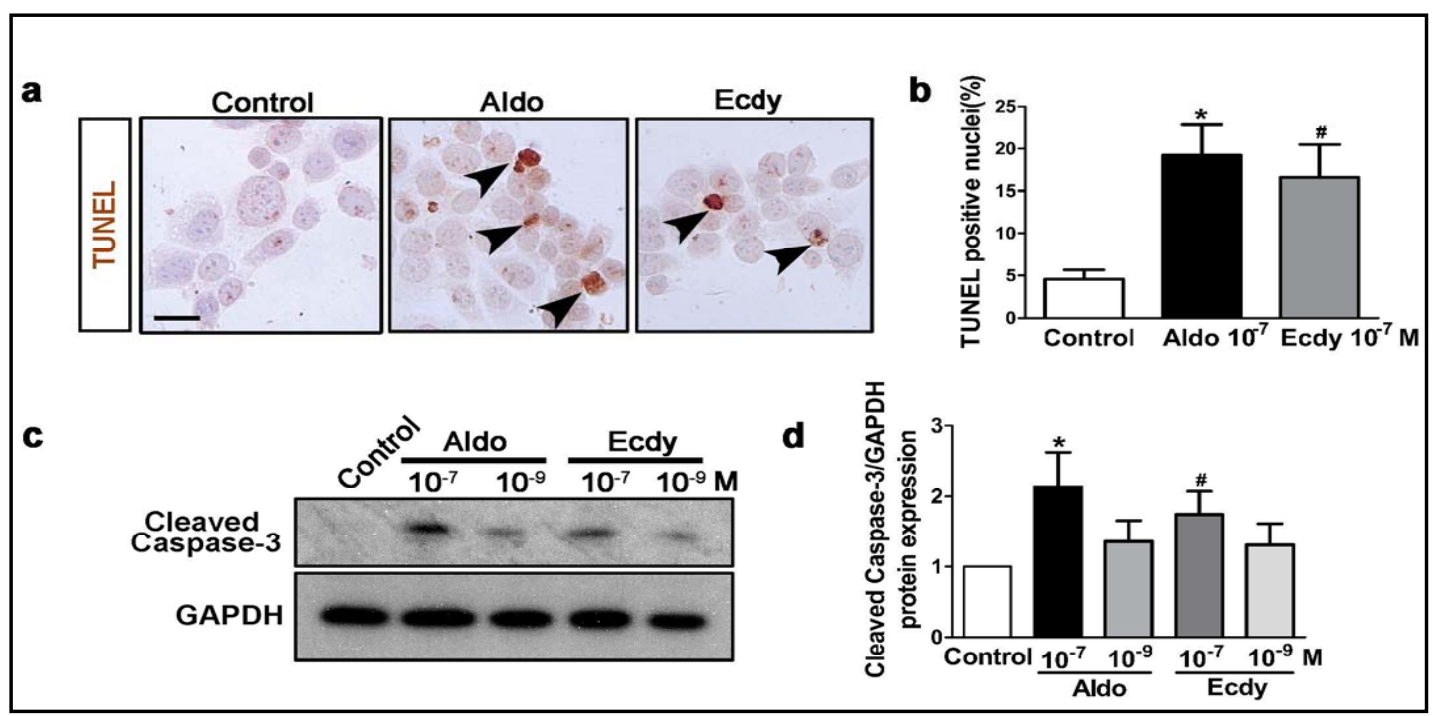

Fig. 4. Ecdysone and aldosterone demonstrate a comparable pro-apoptotic effect on IMCD cells. (A) Cells were treated as elaborated in Fig. 2. Cells were fixed at $48 \mathrm{~h}$ after indicated treatments and processed for TdT-mediated dUTP nick end labeling (TUNEL) staining for apoptotic cells (arrowheads). Bar=20 $\mu \mathrm{m}$. (B) Absolute counting of the numbers of TUNEL-positive cells. Results are presented as the means of percent of TUNEL-positive cells in 10 random high-power fields. ${ }^{*} \mathrm{P}=0.001$ vs control, $\mathrm{P}=0.004$ vs control $(n=3)$. (C) Cell lysates were collected at $48 \mathrm{~h}$ and processed for western immunoblot analysis for indicated proteins. (D) Immunoblots were subjected to densitometric analysis of the relative levels of expression as normalized to GAPDH. * $\mathrm{P}=0.002$ vs control, ${ }^{\#} \mathrm{P}=0.019$ vs control $(\mathrm{n}=3)$.

prediction, which did not identify MR as a target of 20E (data not shown). Thus, Ecdysone, like aldosterone, but not 20E, stimulates several mesenchymal-associated phenotypes in cultured renal tubular cells. 


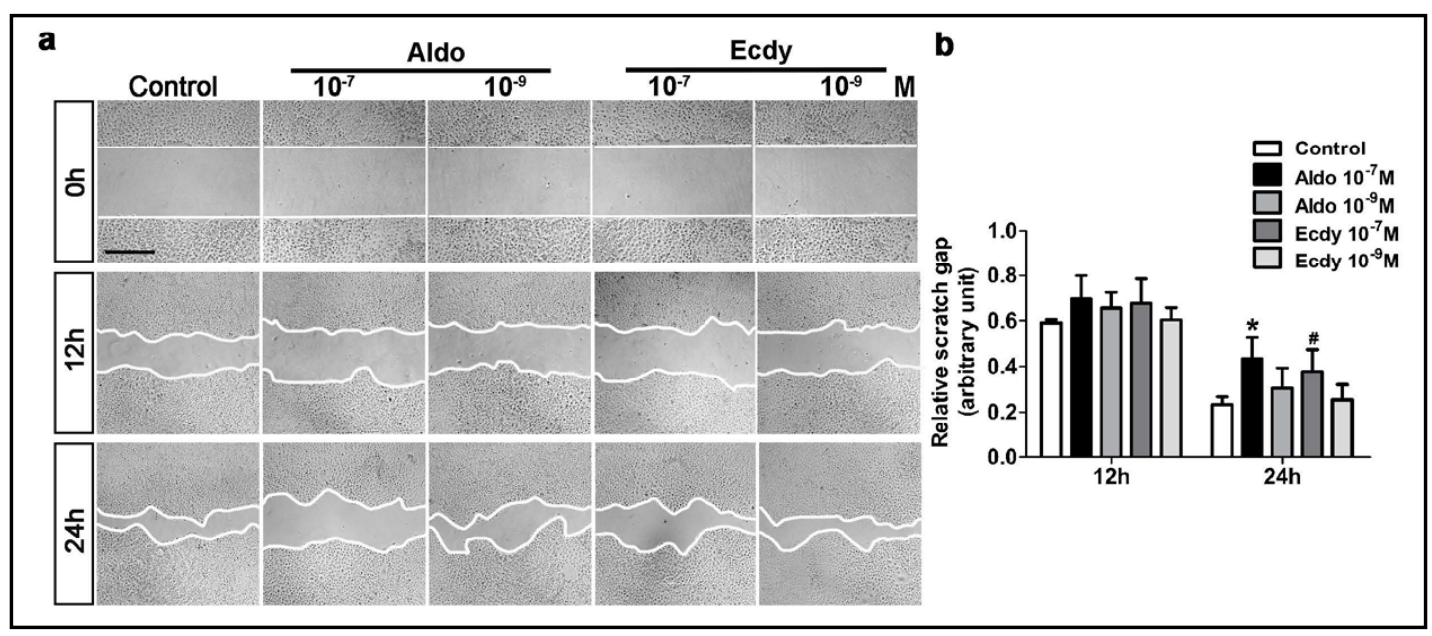

Fig. 5. Ecdysone and aldosterone similarly retard cellular motility in renal tubular cells. (a) Confluent monolayers of IMCD cells were scraped with a $10 \mu \mathrm{l}$ pipette and visualized at 0,12 or $24 \mathrm{~h}$ after ecdysone (Ecdy) or aldosterone (Aldo) treatments using a phase contrast microscope. Representative micrographs were shown. The leading edges of the migrating cell sheets were indicated by the white lines. Bar $=200 \mu \mathrm{m}$. (b) The gap between the invading fronts of the cells was measured by computerized morphormetric analysis. ${ }^{*} \mathrm{P}=0.001$ vs control, ${ }^{*} \mathrm{P}=0.01$ vs control $(\mathrm{n}=3)$.

Both ecdysone and aldosterone similarly cause renal tubular cell apoptosis

Aldosterone at supraphysiological dose is known to cause cell death in vivo and in vitro via a number of mechanisms, including oxidative stress [31]. Here, in cultured IMCD cells, treatment with high dose aldosterone $\left(10^{-7} \mathrm{M}\right)$ for $48 \mathrm{~h}$ induced cellular apoptosis as determined by TUNEL staining. Ecdysone treatment at the same concentration produced a similar pro-apoptotic effect (Fig. 4a, b). Cell lysates were then prepared after different treatments and processed for immunoblot analysis for cleaved caspase 3, a proteolytic enzyme crucial for the execution of apoptosis. Both Aldosterone and ecdysone evidently elevated the expression of cleaved caspase 3 in a dose dependent fashion (Fig. 4c, d).

\section{Ecdysone impedes cellular motility in renal tubular cells}

Aldosterone is known to affect cellular motility and regulate wound healing in both in vivo and in vitro models [32]. Here we used a scratch-wound cell migration assay to examine if ecdysone has a similar effect on cellular motility. IMCD cells under basal conditions readily migrated at leading edges after the monolayer was disrupted (Fig. 5a). The capacity to migrate was reduced by aldosterone in a dose dependent manner, as measured by delay in gap closure. Ecdysone at the same dose suppressed cell motility and scratch wound closure to a comparable extent. Digital morphometric measurements of cell migration area quantified these observations (Fig. 5b).

\section{Ecdysone triggers MR activation in renal tubular cells in vivo and in vitro}

The aldosterone effects noted above are conveyed, at least in part, via activation of the nuclear receptor and transcription factor MR [33]. MR in IMCD cells was therefore examined to determine if ecdysone requires this nuclear hormone receptor to modulate its aldosteronelike phenotypes. Treated cells were fixed and subjected to fluorescent cytochemistry staining for MR nuclear localization. Under basal condition MR was distributed mainly in the cytoplasm, while aldosterone triggered MR nuclear accumulation (Fig. 6a). These cytological observations were corroborated by immunoblot analysis of nuclear extracts, which revealed a strong induction of nuclear MR by aldosterone treatment (Fig. 6c). As expected, nuclear localization of MR with aldosterone was blunted by concomitant treatment with spironolactone, a highly selective antagonist of MR. In cells treated with ecdysone, nuclear

\section{KARGER}


entry of MR was observed to an extent comparable to that found in aldosterone-treated cells, as estimated by both cytochemistry (Fig. 6a) and immunoblot analysis of nuclear fractions (Fig. 6c).

To determine if ecdysone activates MR in the kidney as known for aldosterone, mice were treated daily with subcutaneous injection of ecdysone or aldosterone at a dose of $6 \mu \mathrm{g} /$ $\mathrm{g} / \mathrm{d}$. After 2 weeks treatment, mice were sacrificed and kidney specimens were processed for peroxidase immunohistochemistry staining to identify nuclear MR. Kidney tissues from control mice displayed minimal nuclear MR, which was located mainly within distal tubules and collecting ducts (Fig. 6b). Ecdysone treatment increased the number of MRpositive renal tubules and enhanced the intensity of MR staining. This effect was abrogated in a subgroup of mice concomitantly treated with spironolactone, suggesting that ecdysone, like aldosterone, acts through nuclear translocation of MR. The immunohistochemistry findings were corroborated by immunoblot analysis of nuclear fractions of whole kidney homogenates (Fig. 6d).

\section{Ecdysone confers an MR dependent nephropathic effect}

Kidney specimens from mice following ecdysone, aldosterone and spironolactone treatment were further examined to assess how ecdysone impacts renal pathology. We previously reported how aldosterone elicits renal pathologic lesions in the absence of systemic hemodynamic disturbance like hypertension [34]. Here, ecdysone treatment for 2 weeks also caused early mild accumulation of extracellular matrix in renal

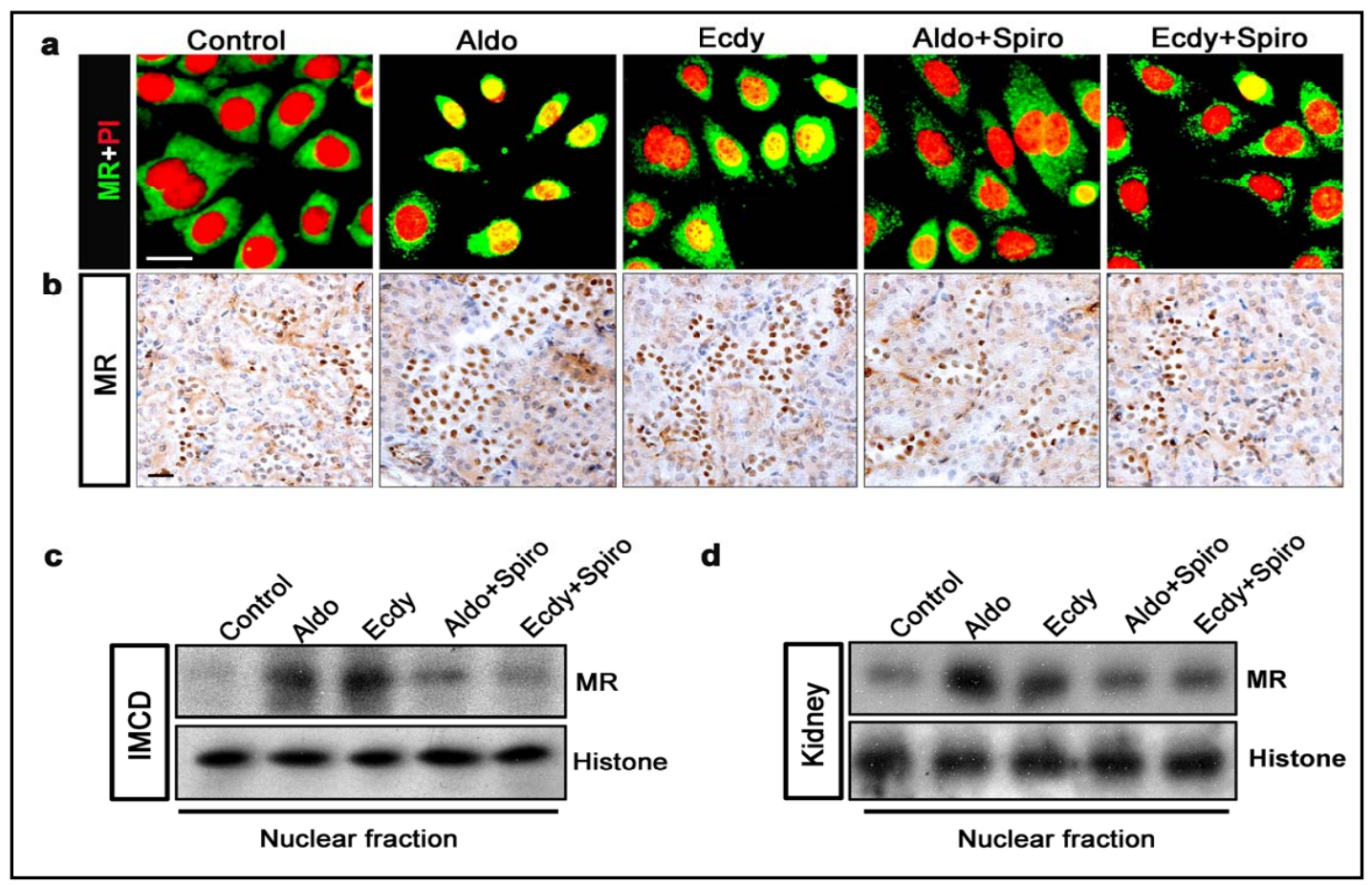

Fig. 6. Ecdysone is capable of activating MR in renal tubular cells in vivo and in vitro. (a) Cells were treated as elaborated in Fig. 2 with ecdysone (Ecdy, $10^{-7} \mathrm{M}$ ) or aldosterone (Aldo, $10^{-7} \mathrm{M}$ ) in the presence or absence of spironolactone (Spiro, $10^{-5} \mathrm{M}$ ). Cells were fixed at 30 minutes after indicated treatments and processed for fluorescence immunocytochemistry staining for minerocorticoid receptor (MR) with propidium iodide (PI) counterstaining of nuclei. Representative micrographs were shown. Bar $=20 \mu \mathrm{m}$. (b) Mice were treated with aldosterone $(6 \mu \mathrm{g} / \mathrm{g} / \mathrm{d})$ or ecdysone $(6 \mu \mathrm{g} / \mathrm{g} / \mathrm{d})$ in the presence or absence of spironolactone $(60 \mu \mathrm{g} / \mathrm{g} / \mathrm{d})$. After 2 weeks, mice were sacrificed and kidney specimens processed for peroxidase immunohistochemistry staining for MR. Representative micrographs were shown. Bar $=20 \mu \mathrm{m}$. (c, d) Nuclear fractions were prepared from IMCD cells and mouse kidney tissues, and then subjected to western immunoblot analysis for MR or histone which served as a loading control for protein normalization. 


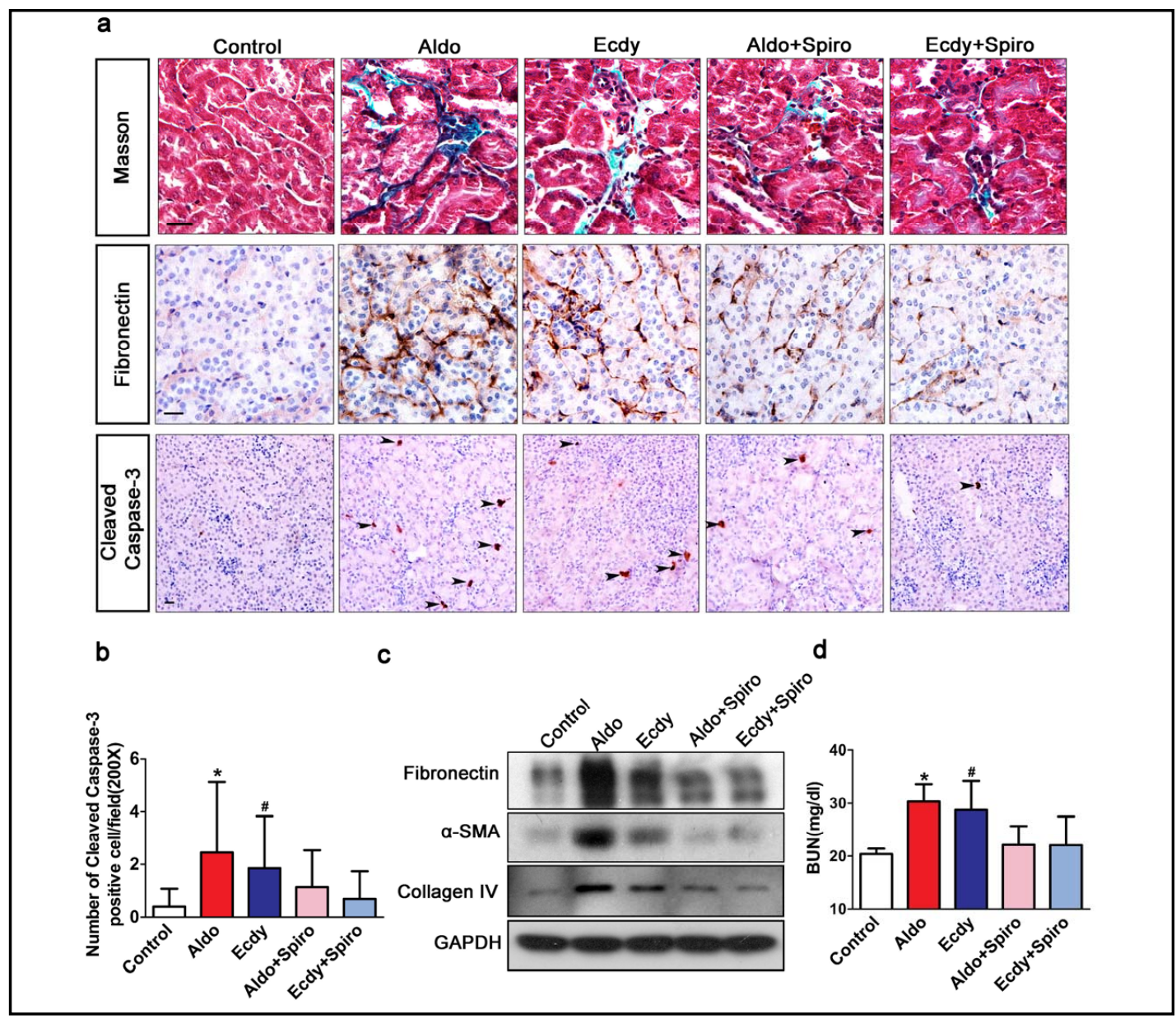

Fig. 7. MR signaling pathway is essential for the nephropathic effects of ecdysone. (a) Mice were treated as described in Fig. 6b. Mouse kidney specimens were processed for Masson trichrome staining or for peroxidase immunohistochemistry staining for fibronectin or cleaved caspase-3. Representative micrographs were shown. Bars $=20 \mu \mathrm{m}$. (b) Kidney homogenates were prepared and subjected to immunoblot analysis for indicated proteins. (c) Absolute counting of the numbers of cells positive for cleaved caspase-3 in kidney sections stained in (a). Results are presented as the means of the number of positive cells in 20 random high-power fields per group. ${ }^{*} \mathrm{P}=0.031$ vs control group; ${ }^{\mathrm{P}} \mathrm{P}=0.047$ vs control group. ( $\left.\mathrm{n}=3 \sim 4\right)$. (d) Plasma samples were processed for blood urea nitrogen (BUN) assay; ${ }^{*} \mathrm{P}<0.05$ vs Control or Aldo+Spiro; ${ }^{\mathrm{P}}<0.05$ vs Control or Ecdy+Spiro ( $n=3 \sim 4)$.

tubulointerstitium, as revealed by Masson trichrome staining (for collagen) and by peroxidase immunohistochemistry staining for fibronectin (Fig. 7a). In addition, ecdysone treatment amplified renal expression of $\alpha$-SMA, a marker for both renal tubular cell dedifferentiation and fibroblast cell activation. Moreover, renal cell apoptosis was considerably augmented in ecdysone-treated animals based on peroxidase immunohistochemistry staining for cleaved caspase 3, which was further quantified by absolute counting of positive cells in a blinded fashion (Fig. 7a, 7b). Histologic observations were corroborated by immunoblot analysis of whole kidney homogenates for collagen IV and fibronectin (Fig. 7c). In concert with the histologic changes, kidney function was evidently impaired after ecdysone treatment, marked by a statistically significant increase in BUN levels (Fig. 7d). The effects of ecdysone were reminiscent of those of aldosterone and substantially attenuated by spironolactone co-treatment, as occurred in ecdysone or aldosterone plus spironolactone-treated animals (Fig. 7a-d). Moreover, the nephropathic effect elicited by ecdysone is likely slow and chronic, akin to aldosterone effect. During our study, no noticeable changes in physical activity, hair

\section{KARGER}




\section{Cellular Physiology Cell Physiol Biochem 2018;49:1633-1645

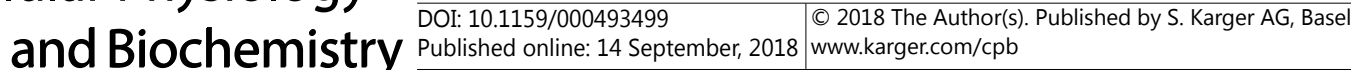

Lu et al.: Harmful Effects of Ecdysone on the Kidney

loss or appetite were observed in mice following ecdysone treatment, consistent with the fact that most clinical or experimental chronic kidney diseases are asymptomatic except for abnormal urinalysis or impaired kidney function.

\section{Discussion}

Contrary to the general belief that natural products used by alternative medicine practices have a great safety profile, a number of natural medications such as aristolochia, licorice and others have recently been found to be potentially harmful for kidney health [3538]. Here we demonstrate that the insect molting hormone ecdysone, which is the major ingredient of common adaptogens, possesses an aldosterone-like activity that is able to activate MR in vitro in aldosterone-responsive cells and in vivo in the kidney. As probably the most abundant steroids in nature, ecdysteroids are not only produced by arthropods but also widely exist in many plant species [4]. It has been popularly used as adaptogens or as anabolic compounds at high doses and for extended durations [6]. In the present study, treatment with high dose ecdysone for only 2 weeks is sufficient to cause early signs of chronic renal impairments in mice. To the best of our knowledge, this is the first study to reveal a nephropathic effect of ecdysone.

The aldosterone-like activity of ecdysone has been described by previous work in other natural substances such as licorice, which is an extract from the root of Glycyrrhiza glabra that contains glycyrrhizic acid and that is widely applied in traditional medicine in many countries [39]. Licorice has been known for many years to be able to cause a mineralocorticoid excess syndrome [40,41]. However, licorice seems to confer aldosterone-like effects not via direct MR activation: the affinity of glycyrrhizic acid for MR is less than 0.01 percent of that of aldosterone [40]. Instead, glycyrrhizic acid inhibits $11 \beta$-hydroxysteroid dehydrogenase (11 $\beta$-HSD) and thus blocks $11 \beta$-HSD catalysis of active cortisol to inactive cortisone. Excess cortisol subsequently over-activates MR-mediated signaling. In contrast, ecdysone appears to activate MR directly, as shown in this study by several lines of evidence. First, in silico analysis based on SciFinder suggested that ecdysone is highly analogous in molecular composition and structure to aldosterone, the cognate ligand of MR. Second, MR ranks as one of the top matched biological targets for ecdysone. The predicted feature of ecdysone binding to MR is highly similar to that of aldosterone: both are modeled to bind to the same LBD domain of MR. Thirdly, in cultured IMCD cells that are known to be quintessential effector cells of aldosterone, ecdysone elicited MR nuclear localization, and induced multifaceted cellular effects that mimicked aldosterone, including mesenchymal-transformation, fibrogenic and apoptotic phenotypes, and retarded cellular motility in a wound assay. Finally, high dose ecdysone treatment of mice caused MR activation within renal tubules, associated with early histologic signs of renal impairment including extracellular matrix accumulation, fibroblast cell activation and apoptosis. These ecdysone-induced effects were largely abolished by simultaneous treatment with spironolactone, a specific MR antagonist. Taken together, we conclude that ecdysone produces mineralocorticoid like action by through the action of MR.

Although the MOE-based 3D molecular modeling and simulations in this study predicted that ecdysone targets the LBD domain of MR, as occurred with aldosterone, future binding analysis is required to validate whether ecdysone might act directly as a ligand to MR. Within invertebrates ecdysone has low affinity for the nuclear hormone receptor EcR $\left(\mathrm{pIC}_{50}=4.7\right.$ ) and does not serve directly as a regulatory ligand [42]. Rather, ecdysone is converted to $20 \mathrm{E}$ within target cells, and $20 \mathrm{E}$ binds to $\mathrm{EcR}\left(\mathrm{pIC}_{50}=6.6\right)$. In contrast to this paradigm, we found that 20E produced no phenotypes in our cellular assays while ecdysone itself was strikingly potent. If ecdysone indeed binds to MR, its structure must uniquely mimic an activating feature of aldosterone that is absent from 20E, where ecdysone and 20E differ only in the addition of a hydroxyl group at C-20. Structural analyses of these subtle differences relative to the MR ligand-binding domain could produce unexpected insights on aldosteroneMR interactions and compounds for renal-related diseases. Alternatively, ecdysone might 
modulate pathology and MR activity through indirect interactions involving alternative receptors and non-genomic signaling [23].

In addition to the MR signaling pathway, ecdysone may impair kidney homeostasis via other mechanisms, as denoted by the residual histologic signs of renal impairment in mice co-treated with the MR blockade spironolactone. The most well-known effect of ecdysone is probably its hypertrophic and anabolic effect that increases protein synthesis in diverse organ systems in rodents and humans, including liver, kidney and most strikingly in muscles [8]. Ecdysone has been verified to be one of the major active ingredients in numerous dietary supplements that are marketed as "natural anabolic agents" and are used by sportsmen or bodybuilders [43]. However, kidney hypertrophy is known to be a prelude to progressive chronic kidney injury. This has been proven in both human patients and animal models in a number of disease states, including diabetic nephropathy, remnant kidney disease, hypokalemic nephropathy, chronic metabolic acidosis and high dietary protein intake [4446]. Anabolic agents are known to lead to kidney hypertrophy and have been shown to impair kidney health and cause chronic kidney disease [47, 48]. Therefore, it is conceivable that ecdysone may cause chronic kidney injury also via its anabolic activities. Future in-depth studies are required to decipher the exact role of the anabolic or hypertrophic activity and its contribution in ecdysone elicited chronic renal impairments.

Our study is not without limitations. It is uncertain how our experimental doses of ecdysone relate to titers from supplements used by humans. As well, we report short-term responses to ecdysone while human supplementation regimes can be extensive and chronic. We demonstrate early signs of chronic kidney impairment in our models but do not yet know how ecdysone affects physiological kidney function or advanced progressive chronic kidney disease. Long-term study is also merited for exploring possible substantive effects of ecdysone on glomerular and tubular pathobiology. Yet even at this stage, our data clearly demonstrate a potential harmful effect of ecdysone on kidney health, and in many ways this insect steroid mimics many features of chronic, elevated aldosterone.

\section{Conclusion}

In summary, our study demonstrated that ecdysone is highly similar to aldosterone in molecular structures and potential biological targets. In cultured IMCD cells and in vivo in mice, ecdysone resembles the action of aldosterone and is able to activate MR in renal tubular cells and elicit early signs of chronic kidney impairments, including kidney cell apoptosis and fibrogenesis. Our data suggest that ecdysteroids, the insect steroid hormone, popularly used as adaptogens or for the purpose of bodybuilding, seem to have a renal injurious activity. Our findings may help raise public awareness about the potential adverse effects of ecdysone.

\section{Acknowledgements}

The research work of the authors was supported in part by the National Natural Science Foundation of China grant 81770672, 81670663 and U1604284, the National Key R\&D program of China (2016YFC1305404), the U.S. National Institutes of Health grant DK092485 and DK114006 and the U.S. NIDDK Diabetic Complications Consortium (DiaComp, www. diacomp.org) grant DK076169. We also acknowledge support from the Dean of Biology and Medicine and a SEED Award at Brown University and an incentive fund from the University of Toledo. The funders had no role in the design and conduct of this study, collection and interpretation of the data, or preparation and approval of the manuscript. 


\section{Cellular Physiology Cell Physiol Biochem 2018;49:1633-1645

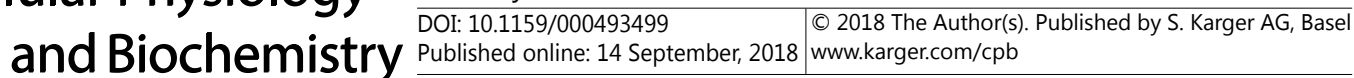 \\ Lu et al.: Harmful Effects of Ecdysone on the Kidney}

\section{Disclosure Statement}

No potential conflicts of interest were disclosed.

\section{References}

1 Panossian A: Understanding adaptogenic activity: specificity of the pharmacological action of adaptogens and other phytochemicals. Ann N Y Acad Sci 2017;1401:49-64.

2 Thiem B, Kikowska M, Maliński MP, Kruszka D, Napierała M, Florek E: Ecdysteroids: production in plant in vitro cultures. Phytochem Rev 2017;16:603-622.

3 Speranza A: Into the world of steroids: a biochemical "keep in touch" in plants and animals. Plant Signal Behav 2010;5:940-943.

4 Tarkowska D, Strnad M: Plant ecdysteroids: plant sterols with intriguing distributions, biological effects and relations to plant hormones. Planta 2016;244:545-555.

5 Dinan L: Phytoecdysteroids: biological aspects. Phytochemistry 2001;57:325-339.

6 Bathori M, Toth N, Hunyadi A, Marki A, Zador E: Phytoecdysteroids and anabolic-androgenic steroids-structure and effects on humans. Curr Med Chem 2008;15:75-91.

7 Bathori M, Pongracz Z: Phytoecdysteroids--from isolation to their effects on humans. Curr Med Chem 2005;12:153-172.

8 Dinan L: The Karlson Lecture. Phytoecdysteroids: what use are they? Arch Insect Biochem Physiol 2009;72:126-141.

-9 Jha V: Herbal medicines and chronic kidney disease. Nephrology (Carlton) 2010;15 Suppl 2:10-17.

10 Jha V, Rathi M: Natural medicines causing acute kidney injury. Semin Nephrol 2008;28:416-428.

11 Perazella MA: Renal vulnerability to drug toxicity. Clin J Am Soc Nephrol 2009;4:1275-1283.

12 Gautam NK, Tapadia MG: Ecdysone signaling is required for proper organization and fluid secretion of stellate cells in the Malpighian tubules of Drosophila melanogaster. Int J Dev Biol 2010;54:635-642.

$\checkmark 13$ Charmantier G, Trilles JP: Effect of the antennary glands on ionic regulation, water retention and eventually, moulting in sphaeroma serratum (crustacea, isopoda, flabelliferg). Gen Comp Endocrinol 1977;31:295301.

14 Cheng DM, Kutzler LW, Boler DD, Drnevich J, Killefer J, Lila MA: Continuous infusion of 20-hydroxyecdysone increased mass of triceps brachii in C57BL/6 mice. Phytother Res 2013;27:107-111.

15 Syrov VN, Kurmukov AG: Anabolic activity of phytoecdysone-ecdysterone isolated from Rhaponticum carthamoides (Willd.) Iljin. Farmakol Toksikol 1976;39:690-693.

16 Masuoka M, Orita S, Shino A, Matsuzawa T, Nakayama R: Pharmacological studies of insect metamorphosing hormone: ponasterone A, ecdysterone, and inokosterone, in the rat. Jpn J Pharmacol 1970;20:142-156.

17 Yamanaka N, Rewitz KF, O'Connor MB: Ecdysone control of developmental transitions: lessons from Drosophila research. Annu Rev Entomol 2013;58:497-516.

-18 Petryk A, Warren JT, Marques G, Jarcho MP, Gilbert LI, Kahler J, Parvy JP, Li Y, Dauphin-Villemant C, O'Connor MB: Shade is the Drosophila P450 enzyme that mediates the hydroxylation of ecdysone to the steroid insect molting hormone 20-hydroxyecdysone. Proc Natl Acad Sci U S A 2003;100:13773-13778.

19 Huang X, Warren JT, Gilbert LI: New players in the regulation of ecdysone biosynthesis. J Genet Genomics 2008;35:1-10.

20 Schwedes CC, Carney GE: Ecdysone signaling in adult Drosophila melanogaster. J Insect Physiol 2012;58:293-302.

21 Nakagawa Y, Henrich VC: Arthropod nuclear receptors and their role in molting. FEBS J 2009;276:61286157.

22 No D, Yao TP, Evans RM: Ecdysone-inducible gene expression in mammalian cells and transgenic mice. Proc Natl Acad Sci U S A 1996;93:3346-3351.

23 Lafont R, Dinan L: Practical uses for ecdysteroids in mammals including humans: an update. J Insect Sci 2003;3:7. 


\section{Cellular Physiology Cell Physiol Biochem 2018;49:1633-1645 \begin{tabular}{l|l|l} 
and Biochemistry 10.1159/000493499 & $\begin{array}{l}\text { C) } 2018 \text { The Author(s). Published by S. Karger AG, Basel } \\
\text { www.karger.com/cpb }\end{array}$ \\
\hline
\end{tabular}}

Lu et al.: Harmful Effects of Ecdysone on the Kidney

-24 Trenin DS, Volodin VV, Beikin Ia B, Shlykova AB: The ecdysteroid fraction of the above-ground portion of Serratula coronata L. in the spontaneous E-rosette formation reaction and the agar migration test in vitro. Eksp Klin Farmakol 1996;59:55-57.

25 Dinan L, Lafont R: Effects and applications of arthropod steroid hormones (ecdysteroids) in mammals. J Endocrinol 2006;191:1-8.

-26 Cahlikova L, Macakova K, Chlebek J, Host'alkova A, Kulhankova A, Opletal L: Ecdysterone and its activity on some degenerative diseases. Nat Prod Commun 2011;6:707-718.

27 Mangelsdorf DJ, Thummel C, Beato M, Herrlich P, Schutz G, Umesono K, Blumberg B, Kastner P, Mark M, Chambon P, Evans RM: The nuclear receptor superfamily: the second decade. Cell 1995;83:835-839.

-28 Li C, Ge Y, Dworkin L, Peng A, Gong R: The beta isoform of GSK3 mediates podocyte autonomous injury in proteinuric glomerulopathy. J Pathol 2016;239:23-35.

29 Haldeman M, Vieira B, Winer F, Knutsen LJ: Exploration tools for drug discovery and beyond: applying SciFinder to interdisciplinary research. Curr Drug Discov Technol 2005;2:69-74.

-30 Wang X, Shen Y, Wang S, Li S, Zhang W, Liu X, Lai L, Pei J, Li H: PharmMapper 2017 update: a web server for potential drug target identification with a comprehensive target pharmacophore database. Nucleic Acids Res 2017;45:W356-W360.

31 Patni H, Mathew JT, Luan L, Franki N, Chander PN, Singhal PC: Aldosterone promotes proximal tubular cell apoptosis: role of oxidative stress. Am J Physiol Renal Physiol 2007;293:F1065-1071.

-32 Slight SH, Chilakamarri VK, Nasr S, Dhalla AK, Ramires FJ, Sun Y, Ganjam VK, Weber KT: Inhibition of tissue repair by spironolactone: role of mineralocorticoids in fibrous tissue formation. Mol Cell Biochem 1998;189:47-54.

-33 Funder JW: Aldosterone and Mineralocorticoid Receptors-Physiology and Pathophysiology. Int J Mol Sci 2017;18.pii: E1032.

-34 Brem AS, Morris DJ, Ge Y, Dworkin L, Tolbert E, Gong R: Direct fibrogenic effects of aldosterone on normotensive kidney: an effect modified by 11beta-HSD activity. Am J Physiol Renal Physiol 2010;298:F1178-1187.

35 Luyckx VA: Nephrotoxicity of alternative medicine practice. Adv Chronic Kidney Dis 2012;19:129-141.

-36 Nauffal M, Gabardi S: Nephrotoxicity of Natural Products. Blood Purif 2016;41:123-129.

37 Luciano RL, Perazella MA: Aristolochic acid nephropathy: epidemiology, clinical presentation, and treatment. Drug Saf 2015;38:55-64.

-38 Nazari S, Rameshrad M, Hosseinzadeh H: Toxicological Effects of Glycyrrhiza glabra (Licorice): A Review. Phytother Res 2017;31:1635-1650.

39 Shibata S: A drug over the millennia: pharmacognosy, chemistry, and pharmacology of licorice. Yakugaku Zasshi 2000;120:849-862.

40 Farese RV, Jr., Biglieri EG, Shackleton CH, Irony I, Gomez-Fontes R: Licorice-induced hypermineralocorticoidism. N Engl J Med 1991;325:1223-1227.

41 Ruszymah BH, Nabishah BM, Aminuddin S, Khalid BA: Effects of glycyrrhizic acid on right atrial pressure and pulmonary vasculature in rats. Clin Exp Hypertens 1995;17:575-591.

42 Minakuchi C, Nakagawa Y, Kamimura M, Miyagawa H: Binding affinity of nonsteroidal ecdysone agonists against the ecdysone receptor complex determines the strength of their molting hormonal activity. Eur J Biochem 2003;270:4095-4104.

43 Parr MK, Botre F, Nass A, Hengevoss J, Diel P, Wolber G: Ecdysteroids: A novel class of anabolic agents? Biol Sport 2015;32:169-173.

44 Martin WF, Armstrong LE, Rodriguez NR: Dietary protein intake and renal function. Nutr Metab (Lond) 2005;2:25.

45 Kronenberg F: Emerging risk factors and markers of chronic kidney disease progression. Nat Rev Nephrol 2009;5:677-689.

46 Kopple JD, Kalantar-Zadeh K, Mehrotra R: Risks of chronic metabolic acidosis in patients with chronic kidney disease. Kidney Int Suppl 2005:S21-27.

47 Maravelias C, Dona A, Stefanidou M, Spiliopoulou C: Adverse effects of anabolic steroids in athletes. A constant threat. Toxicol Lett 2005;158:167-175.

48 Herlitz LC, Markowitz GS, Farris AB, Schwimmer JA, Stokes MB, Kunis C, Colvin RB, D’Agati VD: Development of focal segmental glomerulosclerosis after anabolic steroid abuse. J Am Soc Nephrol 2010;21:163-172. 